\section{Presentations of Clinical, Ultrasonographic and Pathological Features of Nodular Fasciitis from an Established Cytogenetic Viewpoint: Review of the Case Series}

\author{
Kazumi Fujioka ${ }^{*}$ \\ Division of Laboratory Medicine, Department of Pathology and Microbiology, Nihon University School of Medicine, Itabashi-ku, Tokyo, Japan
}

*Corresponding author: Dr. Kazumi Fujioka, Division of Laboratory Medicine, Department of Pathology and Microbiology, Nihon University School of Medicine, Itabashiku, Tokyo, Japan; E-mail: fujioka.kazumi@nihon-u.ac.jp

Received date: November 08, 2018; Accepted date: November 15, 2018; Published date: November 19, 2018

Copyright: @ 2018 Fujioka K. This is an open-access article distributed under the terms of the Creative Commons Attribution License, which permits unrestricted use, distribution, and reproduction in any medium, provided the original author and source are credited.

\begin{abstract}
Even though nodular fasciitis (NF) is benign and self-limited, the clinical, ultrasonographyic and pathological presentations have been described as mimicking sarcoma. We have reported that proliferative findings on both US and histology may be caused by the cytogenetic nature of NF. When the lesion showed the proliferative findings of the margin on both US and pathology, accompanied with clinical rapid growth and self-limited course, NF should be strongly suggested as one of the skin tumors as previously described. NF has been cytogenetically considered as a novel model of transient neoplasia induced by MYH9-USP6 gene fusion. It has been suggested that strong overexpression of USP6 under MYH9 promoter appears to drive tumorigenesis. A clear association between NF and a recurrent genetic abnormality had been established in fluorescence in-situ hybridization (FISH) analysis.
\end{abstract}

In this article, we reviewed the clinical, ultrasonographic and pathological features of NF in the case series from an established cytogenetic viewpoint. Our study indicated that proliferative findings of the margin on US and pathology may be caused by the driving force of USP6 transcriptional upregulation behind the high proliferative activity and growth of NF. All nodules clinically represented rapid growing, self-limited and/or regress course, suggesting the high proliferative growth and involutional nature of NF.

We emphasize that the presentations of clinical, ultrasonographic and pathological features of NF are contributed to the cytogenetic nature, having the high proliferative growth and involutional nature.

Keywords: Nodular fasciitis; USP6-induced neoplasia; Highresolution ultrasound; Proliferative growth; Involutional nature

\section{Introduction}

NF is a benign soft tissue tumor of fibroblastic/myofibroblastic differentiation that was first described in 1955 by Konwaler, et al. [1] and Goodlad JR, et al. [2] documented the first case of dermal nodular fasciitis (NF) in 1990. Ordinary NF and intradermal NF show a peculiar clinical behavior characterized by rapid, self-limited growth and spontaneous regression after a few weeks. The aggressive clinical presentation of NF will usually prompt a biopsy for definitive diagnosis [3]. Due to the histopathology with its high cellularity and high mitotic activity, NF can be misdiagnosed as a malignant soft tissue tumor, especially fibrosarcoma or low-grade myxofibrosarcoma, often leading to unnecessarily aggressive therapy [4].

To study the characteristic ultrasonographic features of NF will help to establish preoperative diagnostic criteria and to avoid unnecessary operation. On gray-scale US, a few reports [3] have described the lesion as irregular or lobulated margin and hypoechogenicity. There are also some reports [3,5-8] concerning the imaging features on color Doppler US. The high-resolution ultrasonography (US) is a valuable tool that the discrepancies of imaging feature between intradermal and subcutaneous cases [9]. We have recently reported characteristic power Doppler sonographic imaging of NF from a dermatological perspective [10]. Erickson-Johnson, et al. [11] first have reported that NF is as a novel model of transient neoplasia induced by MYH-USP6 gene fusion. It has been described that a strong overexpression of USP6 under $M Y H 9$ promoter appears to drive tumorigenesis [12]. A clear relation between NF and a recurrent genetic abnormality has been established in FISH analysis [11-16]. We think that proliferative findings on both US and histology may be caused by the cytogenetic nature of NF as previously reported [10]. In this article, we review the case series of NF and emphasize the presentations of clinical, ultrasonographic and pathological characteristics from an established cytogenetic perspective.

\section{Results}

Summary of the pathologically confirmed case series of NF were shown (Table 1). The case series included 1 male patient and 3 female patients (mean age, 36.5 years; range, 21-47 years). Three of the 4 patients presented to their physicians with a history of an enlarging palpable mass. They noted pain at the site of the mass. One patient noted pain in the thigh and she incidentally found mass (case 2) [6]. The size of the incision-biopsied NF was found to greatly diminish to a mere shadow and no longer recognizable mass by follow-up US examination as previously described (case 2) [6]. The mean time to presentation of the 3 patients was 1 month. None of the patients reported a history of trauma. One lesion was located in the upper arm (case 1) [10]. 1 was located in the thigh (case 2) [6], and 1was located in the forearm (case 3) [7] and 1 was located in the dorsum of the foot (case 4). One lesion was located in the subcutaneous tissues (case 1); 1 
Citation: Fujioka K (2018) Presentations of Clinical, Ultrasonographic and Pathological Features of Nodular Fasciitis from an Established Cytogenetic Viewpoint: Review of the Case Series. J Carcinog Mutagen 9: 326. doi:10.4172/2157-2518.1000326

Page 2 of 5

was located in the subcutaneous adjacent of the fascia (case 2); 1 was located in the primarily in the dermis (case 3 ) and 1 was located in the upper portion of the subcutaneous (case 4).

\begin{tabular}{|l|l|l|l|l|l|l|l|l|l|l|l|}
\hline \multicolumn{4}{|l|}{ Clinical features } & \multicolumn{3}{l|}{ Ultrasonographic features } & \multicolumn{3}{l|}{ Immunohistochemical features } \\
\hline Case & Age & Sex & Location & Size & Shape & Margin & SMA & Vimentin & Desmin & S-100 protein \\
\hline 1 & 21 & F & Upper arm & $7.8 \mathrm{~mm}$ & Oval & Irregular and lobular & + & ND & - & ND \\
\hline 2 & 41 & F & Thigh & $17 \mathrm{~mm}$ & Oval & Irregular and lobular & - & + & ND & - \\
\hline 3 & 37 & M & Forearm & $8.0 \mathrm{~mm}$ & Oval & Irregular and lobular & ND & ND & ND & ND \\
\hline 4 & 47 & F & Dorsum of the foot & $8.5 \mathrm{~mm}$ & Oval & Lobular & + & ND & - & ND \\
\hline ND: Not determined & & & & & & & & \\
\hline
\end{tabular}

Table 1: Summary of the pathologically confirmed cases of NF.

On gray-scale US, all nodules were oval shape with irregular and/or lobular margin (Figure $1 \mathrm{~A}$ ), averaging $10.3 \mathrm{~mm}$ (range, $7.8-17 \mathrm{~mm}$ ). Two nodules were hypoechoic pattern and one was hyperechoic containing an irregular hypoechoic area in its center and one was hypoechoic pattern with heperechoic streaks. A heterogeneous echotexture was observed in two cases and homogeneous echotexture in two cases. All cases did not represent posterior echo enhancement and one case represented the compressed fascia. Two cases showed clear blood flow within the nodule and two cases no substantial blood flow on color Doppler US (Figure 1B).

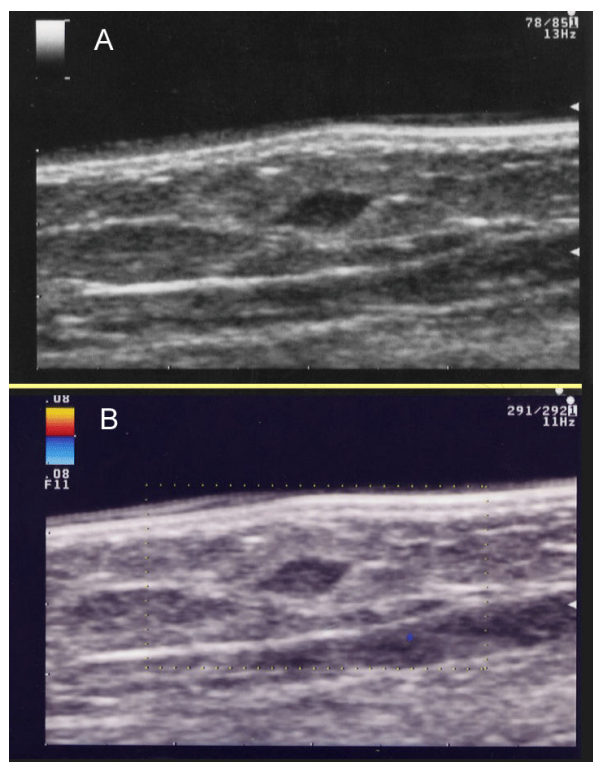

Figure 1: Subcutaneous subtype of NF at the upper arm in a 21year-old woman (case 1). Sonography shows a subcutaneous hypoechogenic lesion located in the upper arm. The shape of nodule is oval with irregular and lobular margin. (B) No substantial blood flow signals within the nodule on color Doppler sonography are observed.

Pathologically, three nodules showed poorly defined margin (Figure 2A) and one represented relatively poorly defined margin.
Proliferations of fibroblast-like spindle cells without obvious mitotic changes were seen in all cases (Figure 2B). All lesions showed myxoid change, collagen proliferations (Figure $3 \mathrm{~A}$ ). Capillary dilatations were seen in all lesions (Figure 3B).

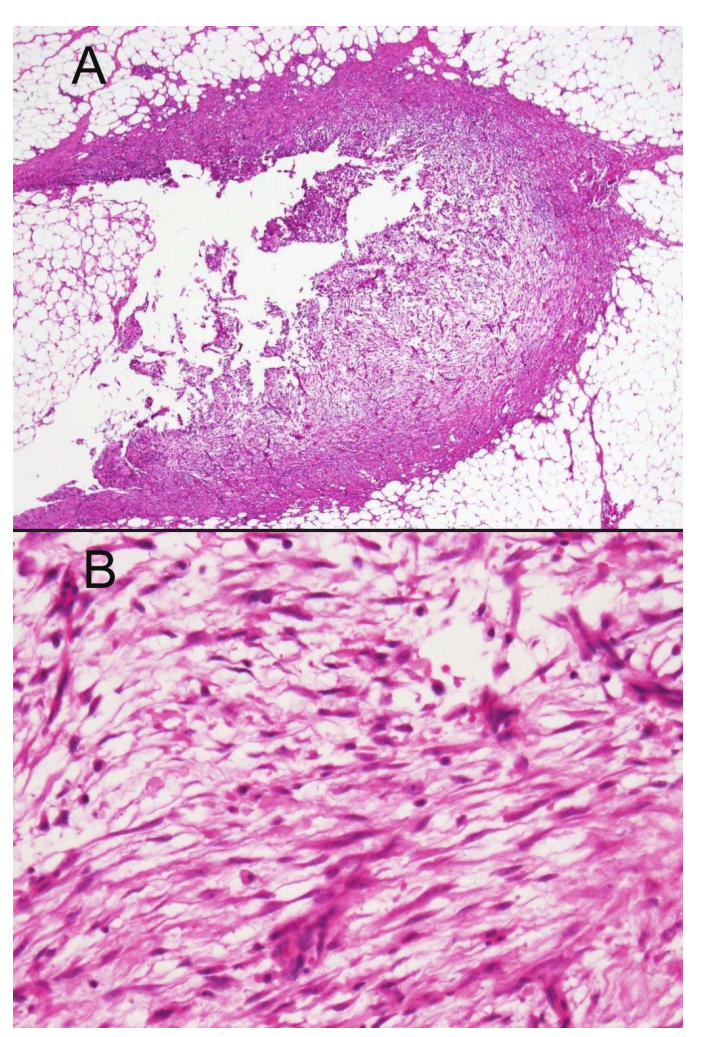

Figure 2: Subcutaneous subtype of NF at the upper arm in a 21year-old woman (case 1). (A) Histopathological finding of the lesion is characterized by the infiltration of fibroblast-like spindle cells in the subcutaneous fatty tissue (hematoxylin and eosin, original magnification $\mathrm{X} 20$ ). (B) The inside of the nodule shows the immature plum fibroblasts arranged in short irregular bundles and fascicles with a feather-like appearance as well as abundant myxoid changes (hematoxylin and eosin, original magnification X200). 


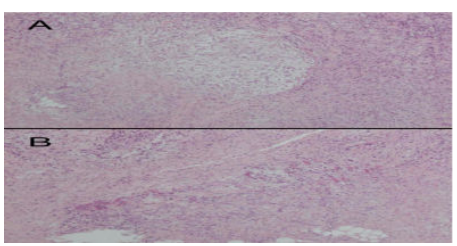

Figure 3: Intradermal subtype of NF at the forearm in a 37-year-old man (case 3). (A) The pathologic examination showed spindleshaped cells resembling fibroblasts and inflammatory cells primarily in the dermis. The inside of the nodule showed the deposition of the mucin in the stroma (hematoxylin and eosin, original magnification X100). (B) The dilatation of blood vessels filled with erythrocytes was observed (hematoxylin and eosin, original magnification X100).

The immunohistochemical staining profile showed smooth muscle actin (SMA) in two cases (Figure 4A) and did not stain for a-SMA in one case. Lesion did not stain for desmin in two cases (Figure 4B). Case 2 showed positivity for vimentin and negative profile for S-100 protein as previously described [6].

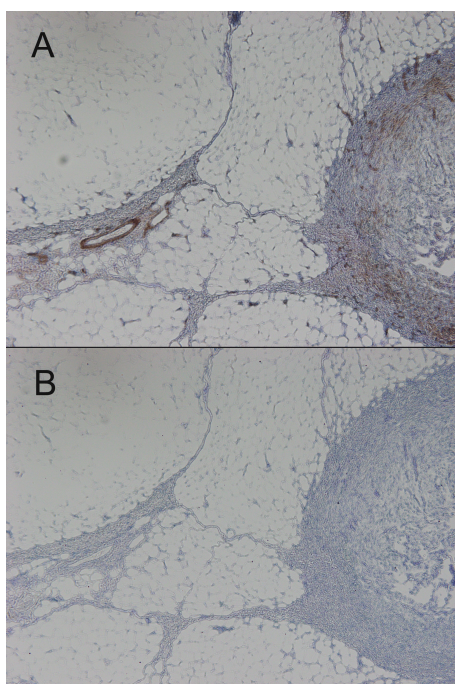

Figure 4: Subcutaneous subtype of NF at the upper arm in a 21year-old woman (case 1). (A) Immunohistochemical finding shows that lesion is positive for smooth muscle actin: original magnification X 100. (B) The lesion is negative for desmin: original magnification X100.

\section{Discussion}

NF presents most typically in the upper extremities, the trunk, and the head and neck. It usually presents between the ages of 20 and 40 years, often with tenderness. Lesions can be found subcutaneous, fascial, intramuscular, and rarely intradermal type. Even though NF is benign and self-limited, the clinical, ultrasonographic and pathological presentations have been described as mimicking sarcoma. To study the characteristic ultrasonographic features of NF will help to establish preoperative diagnostic criteria and to avoid unnecessary operation.
The usefulness of high-resolution US has technically also enabled the discrepancies of imaging features between intradermal and subcutaneous NF $[9,10]$. Meanwhile, NF shows a peculiar clinical behavior characterized by rapid growing, self-limited, spontaneous regression after a few weeks; this peculiar clinical presentation has come to cytogenetic attention. Erickson-Johnson, et al. [10] first has reported that NF is as a novel model of transient neoplasia induced by MYH9-USP6 gene fusion. A clear association between NF and a recurrent genetic abnormality has been established in FISH analysis [11-16]. Clinical presentation showed a few weeks history of a rapid growing mass, self-limited and/or tended to regress with pain in all cases. The size of the incision-biopsied NF (case 2) was found to greatly diminish to a mere shadow and no longer recognizable mass by follow-up US examination as previously described [6]. We will highlight that these features reflect high proliferative activity and growth and involution nature of NF.

On gray-scale US, there are a few reports [3] that the lesions were described as oval or lobulated and hypoechoic or mixed echogenicity with irregular or lobular margin. There are also a few reports [3,5-8] concerning the imaging features on color Doppler US. We have recently reported characteristic power Doppler sonographic imaging of NF from a dermatological perspective [10]. Present study indicated that all nodules showed irregular or lobular margin. No significant different findings between the intradermal and subcutaneous types on gray-scale US were detected. When the US and pathological findings in all cases were compared, irregular or lobular margin on US seemed to reflect histological proliferation of collagen and spindle-shaped fibroblasts. Mehregan [17] has reported that NF is usually composed of a central solid mass with many irregular projections extending outward into the surrounding fat tissue thus resembling a highly invasive growth. These histopathological findings were seen in most of all cases. We suggest that these aspects reflect high proliferative activity and growth of NF as previously described [10]. In this article, we will emphasize that proliferative findings of the margin on US and pathology are caused by the driving force of USP6 transcriptional upregulation behind the high proliferative activity and growth of NF.

Histologically, accurate diagnosis, particularly on small biopsies, remains a challenge, as the morphology can be varied and immunophenotype is essentially nonspecific. Lesions that were considered to be definite diagnosis of NF typically featured uniform spindled cells arranged in irregularly intersecting short fascicles, and occasional storiform patterns. The cells contained plump, spindled to stellate nuclei with fine to open chromatin and small nucleoli, accompanied by lightly basophilic cytoplasm with ill-defined cytoplasmic borders. The background stroma ranged from myxoid to collagenous. The characteristic tissue culture-like, feathery myxoid appearance was present at least focally [13]. As NF closely mimics sarcoma, it is significant that NF differentiate from sarcoma. As sarcoma is composed of spindle cells which are believed to be of myofibroblastic/fibroblastic origin, these tumors have a diffuse inflammatory infiltrate and a variable myxoid stroma without tissue culture growth pattern seen in NF [12].

Immunohistochemically, it has been reported that NF is negative for several markers such as desmin, keratin, or S-100 protein in contrast to sarcoma $[18,19]$. NF spindle cells contain vimentin, muscle-specific actin and smooth muscle-specific actin [19]. On the basis of the stain profile, three cases were immunohistochemically consistent with as a NF. 
A clear association between NF and a recurrent genetic abnormality had been established in FISH analysis [11-16]. Genomic rearrangements of the USP6 locus were found in $92 \%$ of NF. Rapid amplification of 5'-cDNA ends identified $M Y H 9$ as the translocation partner. RT-PCR and direct sequencing revealed the fusion of the $M Y H 9$ promoter region to the entire coding region of USP6 [11]. The MYH9-USP6 fusion was demonstrated in 12 of 16 (75\%) [11]. USP belongs to a large subfamily of de-ubiquitinating enzymes that act in diverse cellular processes such as intracellular trafficking protein turnover, inflammatory signaling and cell transformation. USP6 was first identified as a possible oncogene located on chromosome $17 \mathrm{p} 13$ when it exhibited transforming properties upon overexpression in NIH-3T3 cell [20,21]. USP6 oncogenes was also detected in aneurysmal bone cyst (ABC) $[20,21]$. It has been reported that $A B C$ and NF have overlapping histological features and these histological manifestations may have a common pathogenic denominator mediated by USP6 transcriptional upregulation [11]. Ye Y, et al reported that USP6-induced expression of matrix metalloproteases through activation of NF- $\kappa \mathrm{B}$, a transcription factor with a key role in inflammation and proliferation in $\mathrm{ABC}$ [22]. Oliveira $\mathrm{AM}$ et al. suggested that $\mathrm{ABC}$ and $\mathrm{NF}$ reside in the same biologic spectrum of what they define as USP6-induced tumors [23]. It has been reported that the genomic rearrangements of the USP6 locus were located in $92 \%$ of NF [11] and in $74 \%$ of NF [16]. It has been also reported that in the morphologically definitive cases of NF, FISH analysis for USP6 had a sensitivity of $86 \%$ and specificity of $100 \%$ for a diagnosis of NF and the positive predictive value was $100 \%$, and the negative predictive value $90 \%$ [13]. Erber R, et al. also reported that the sensitivity and specificity of USP6 FISH for NF was $74.4 \%$ and $100 \%$, respectively [14]. These studies indicate a clear relation between NF and recurrent genetic abnormality in FISH analysis.

MYH9 is located on chromosome 22q12.3-q13 and is a member of the non-muscle myosin classfamily [11]. It has been described that $M Y H 9$ is the most frequent fusion partner $[11,16]$. Less frequent gene partners include RRBP1, CALU, CTNNB1, MIR22HG, SPARC, THBS2, and COL6A2 [15]. Their study detected the neoplastic nature of NF, defined additional pathogenic fusion partners and added to the growing body of literature on USP6-associated neoplasia [15].

In cutaneous NF, Kumar, et al. [12] reported USP rearrangement by FISH; in two of three cases, the characteristic MYH9-USP6 fusion was shown by RT-PCR. They concluded that the presence of the USP6 rearrangement and MYH9-USP6 fusion in cutaneous NF cases mirrors what has been reported in more typical subcutaneous presentation of NF. On the basis of these studies [11-16], the similar cytogenetic nature has been demonstrated both in subcutaneous NF and intradermal type.

Erickson-Johnson, et al. [11] suggested that USP6 transcriptional upregulation may be the driving force behind the high proliferative activity and growth of NF. They also noted that the consistent involutional nature of the lesion indicates that additional genetical or stromal factors are required for maintaining its long-term survival and viability. Previous report indicated that irregular or lobular margin on US and poorly defined margin on histology is contributed to the cytogenetic nature of NF [10]. This article strongly suggests that proliferative findings the margin of the nodule on US and pathological features are caused by the driving force of USP6 transcriptional upregulation behind the high proliferative activity and growth of NF from an established cytogenetic nature. All nodules clinically represented rapid growing, self-limited and/or regress, suggesting high proliferative activity and growth and involutional nature of NF. When the lesion showed the proliferative findings of the margin on both US and pathology, accompanied with clinically rapid growth, self-limited and/or regress course, NF could be strongly suggested.

\section{Conclusion}

We emphasize that clinical, ultrasonographic and pathological features of NF are contributed to the cytogenetic nature of the high proliferative growth and involutional nature.

\section{Acknowledgments}

The author deeply appreciates the assistances of Dr. Akira Fujioka, Dr. Minoru Oishi, Dr. Ken Hayashi, Dr. Hikaru Eto and Dr. Tomohiro Nakayama.

\section{References}

1. Konwaler BE, Keasbey L, Kaplan L (1955) Subcutaneous pseudosarcomatous fibromatosis (fasciitis). Am J Clin Pathol 25: 241-252.

2. Goodlad JR, Fletcher CD (1990) Intradermal variant of nodular 'fasciitis'. Histopathol 17: 569-571.

3. Khuu A, Yablon CM, Jacobson JA, Inyang A, Lucas DR, et al. (2014) Nodular Fasciitis: Characteristic imaging features on sonography and magnetic resonance imaging. J Ultrasound Med 33: 565-573.

4. Nishio J (2013) Updates on the cytogenetics and molecular cytogenetics of benign and intermediate soft tissue tumors. Oncol Lett 5:12-18.

5. Nikolaidis P, Gabriel HA, Lamba AR, Chan NG (2006) Sonographic appearance of nodular fasciitis. J Ultrasound Med 25: 281-285.

6. Fujioka K, Fujioka A, Eto H, Suzuki K, Sanuki E, et al. (2006) Nodular fascitis in the thigh followed up using ultrasonography. J Med Ultrason 33: 49-53.

7. Fujioka K, Fujioka A, Oishi M, Eto H, Tajima S, et al. (2017) Ultrasonography findings of intradermal nodular fasciitis: a rare case report and review of the literature. Clin Exp Dermatol 42: 335-336.

8. Lee KJ, Jin W, Kim GY, Rhee SJ, Park SY, et al. (2015) Sonographic features of superficial-type nodular fasciitis in the musculoskeletal system. J Ultrasound Med 34:1465-1471.

9. Wortsman X (2012) Common applications of dermatologic sonography. J Ultrasound Med 31: 97-111.

10. Fujioka K, Fujioka A, Tajima S, Oishi M, Hayashi K, et al. (2018) Characteristic power Doppler sonographic imaging of nodular fasciitis from a dermatological perspective: another case and review of three cases. J Clin Case Rep 8: 1165.

11. Erickson-Johnson MR, Chou MM, Evers BR, Roth CW, Seys AR, et al. (2011) Nodular fasciitis: a novel model of transient neoplasia induced by MYH9-USP6 gene fusion. Lab Invest 91: 1427-1433.

12. Kumar E, Patel NR, Demicco EG, Bovee JV, Olivera AM, et al. (2016) Cutaneous nodular fasciitis with genetic analysis: a case series. J Cutan Pathol 43: 1143-1149.

13. Shin C, Low I, Ng D, Oei P, Miles C, et al. (2016) USP6 gene rearrangement in nodular fasciitis and histological mimics. Histopathology 69: 784-791.

14. Erber R, Agaimy A (2018) Misses and near misses in diagnosing nodular fasciitis and morphologically related reactive myofibroblastic proliferations: experience of a referral center with emphasis on frequency of USP6 gene rearrangements. Virchows Arch 473: 351-360.

15. Patel NR, Chrisinger JSA, Demicco EG, Sarabia SF, Reuther J, et al. (2017) USP6 activation in nodular fasciitis by promoter-swapping gene fusions. Mod Pathol 30: 1577-1588.

16. Amary MF, Ye H, Berisha F, Tirabosco R, Presneau N, et al. (2013) Detection of USP6 gene rearrangement in nodular fasciitis: an important diagnostic tool. Virchows Arch 463: 97-98. 
Citation: Fujioka K (2018) Presentations of Clinical, Ultrasonographic and Pathological Features of Nodular Fasciitis from an Established Cytogenetic Viewpoint: Review of the Case Series. J Carcinog Mutagen 9: 326. doi:10.4172/2157-2518.1000326

Page 5 of 5

17. Mehregan AH (1966) Nodular fasciitis. Arch Dermatol 93: 204-210.

18. Lloyd AA, Witheiler D, Menter A (2015) Nodular fasciitis of the lip mucosa: a rare but clinically important entity. Clin Exp Dermatol 40: 408-412.

19. Borumandi F, Cascarini L, Mallawaarachchi R, Sandison A (2012) The chameleon in the neck: nodular fasciitis mimicking malignant neck mass of unknown primary. International J Surgery Case Rep 3: 501-503.

20. Oliveira AM, Perez-Atayde AR, Inwards CY, Medeiros F, Derr V, et al. (2004) USP6 and CDH11 oncogenes identify the neoplastic cell in primary aneurysmal bone cysts, and are absent in so-called secondary aneurysmal bone cysts.. Am J Pathol 165:1773-1780.
21. Oliveira AM, Perez-Atayde AR, Dal Cin P, Gebhardt MC, Chen CJ, et al. (2005) Aneurysmal bone cyst variant translocations upregulate USP6 transcription by promoter swapping with the ZNF9, COL1A1, TRAP150, and OMD genes. Oncogene 24: 3419-3426.

22. Ye Y, Pringle LM, Lau AW, Riquelme DN, Wang H, et al. (2010) TRE17/ USP6 oncogene translocated in aneurysmal bone cyst induces matrix metalloproteinase production via activation of NF-kappaB. Oncogene 29: 3619-3629.

23. Oliveira AM, Chou MM (2014) USP6-induced neoplasms: the biologic spectrum of aneurysmal bone cyst and nodular fasciitis. Hum Pathol 45: $1-11$. 